\title{
Hybrid Artificial Bee Colony Based Feature Selection in Bone Marrow Plasma Cell Gene Expression Data
}

\author{
Ragunthar. $\mathrm{T}^{\mathbf{1}}$, Selvakumar. $\mathrm{S}^{\mathbf{2}}$ \\ ${ }^{1}$ Assistant Professor Senior, School of Computer Science and Engineering, \\ Vellore Institute of Technology, Vellore, Tamilnadu , India. \\ ragunthar.t@ vit.ac.in \\ ${ }^{2}$ Professor, Department of Computer Science and Engineering, \\ G K M college of Engineering and Technology, Chennai, Tamilnadu, India. \\ sselvakumar@yahoo.com
}

\begin{abstract}
The microarray technology permits simultaneous monitoring of several thousand gene expressions for each sample. However, classifying such samples into their distinct classes may be quite challenging owing to the higher number of genes (the features) over the actual samples. As a consequence, there is a need to make an investigation of the new and the robust techniques of machine learning that can accurately classify the microarray data. The B cells further give some humoral immunity by means of distinguishing into the Plasma Cells (PCs) that are antibody-secreting which is a process that needs a cellular division linked to the DeoxyRibonucleic Acid (DNA) hypomethylation. Classification of cancer is another critical issue in the analysis of cancer treatment. A very effective method in the classification of cancer is its gene selection. But choosing the gene subset that increases the accuracy may be a Non-Deterministic Polynomial (NP)-hard problem. For this work, a methodology was proposed for choosing an ideal set of genes which permit classification of the class of disease based on gene expression along with a good accuracy. The proposed method was compared with feature selection method that employed the Genetic Algorithm (GA).The feature selection of the proposed work is using the Artificial Bee Colony (ABC) algorithm with feature selection that is Levy search based. The results have proved that this new technique had an increase in accuracy of classification which was compared to the Genetic Algorithm and also the Artificial Bee Colony based technique. This also had a very fast convergence.
\end{abstract}

Key words : Artificial Bee Colony (ABC), Feature selection, Genetic Algorithm (GA), Gene Expression Data, Gene Selection and Microarray Data.

\section{INTRODUCTION}

The samples of the DNA microarray were generated through mRNA hybridization derived from the tissue sample or the blood and also the cDNA (for a spotted array) and a hybridization of the DNA oligonucleotides (for cases of the Affymetrix chips, this gets made on the chip-array surface). The technology of DNA microarray permits for a instantaneous monitoring along with a measurement of many thousands of gene expressions along with their levels of activation within one experiment. The class memberships have been distinguished by protein production i.e. the gene expressions will consign to the protein level that are produced for a particular gene. This way, a microarray data may be able to provide some expensive results for problems of gene expression and further give to the advancement in medicine. A clinical microarray data application based on classification of the type of cancer has been increasingly gaining popularity recently. Some statistical techniques are coupled with the patterns of gene expression and used for screening the potential markers of tumours. There are some differential expressions of the genes which are analyzed in a statistical manner and these genes will be assigned to different classes that can or at times cannot enhance an understanding of their biological process [1].

A technology of microarray gene expression has recently unlocked the chances of examining the genes along with their activity in a simultaneous fashion. These profiles of gene expression may have shown a measurement of their relative abundance for the mRNA that corresponds to the genes. This way, there can be a discriminant analysis of such microarray data which may have a high potential to serve as a tool for medical analysis. The primary goal of this classification of microarray data was to construct an effective and proficient model to identify genes that have been expressed differentially and used for the prediction of a class membership for various unidentified samples. These confronts that were created in a microarray classification were some restricted dimension of such samples and have been compared to its high dimensionality with some experimental deviations in the gene expression levels [2]. 
Even though there have been several steps found in a B-Cell (BC) development that were all elucidated, the Plasma Cell (PC) and its differentiation in the final stages were not understood well. The PCs that were generated during the time of humoral immune responses had variation within light regions of the original centers that were a part of the lymph nodes or a part of the spleen red pulp with a life-span of some days. At the hour of some humoral invulnerable reactions which were optional, the long-lived PCs tend to travel from their secondary lymphoid tissues to the inside a lamina propria of mucosa or inside a bone marrow within which are able to survive and also secrete plenty of immunoglobulin for a minimum of 3 weeks. In spite of the morphologic or the useful similarities, the PCs are separated from various organs and can display many differences [3].

Multiple Myeloma (MM) has been identified as a tumour of the PCs that are differentiated terminally which is a home to it and also expands within the bone marrow. Even though it appears to be originally from the post-germinal center cell, the presence of somatic hyper-mutation results in speculation regarding the exact cell where this malignant transformation takes place. The MM and its hypo-proliferative nature along with the labelling indexes within the clonal PCs which rarely are more than $1 \%$, has resulted in a hypothesis that an MM tumour from a transformed precursor cell proliferating and differentiating this which results in a new clonal expression of certain mortally distinguished PCs. The bone marrow of the patients of various myelomas further has some BC populations at different stages of their separation which are relayed to the PCs. A microarray analysis of these patterns of global gene expressions is a very powerful way of identification of the clinical subgroups of their hematopoietic neoplasms [4].

There are some conventional approaches for reducing the dimensions in the microarray data which are the feature selection, the individual methods, the specific methods and so on. The relevance value of the gene will be based on its capacity which is expressed differentially. These methods can rank the genes on the basis of their respective relevance used for its discrimination. By means of setting up the threshold, the relevant genes are filtered. There are also two other concepts to feature selection which denote the filters and the wrappers. The former will be data pre-processing or the data filtering. This type of a feature selection was based on their intrinsic characteristics determining relevance as regards the target classes. There have been some more simple methods which are based on their reciprocal information, the arithmetic tests (like the F-test or the t-test) that are efficient. There are some more sophisticated methods developed and the filter methods had been easily and efficiently computed [5].

They are different types of features in digital field like, The image feature like color, texture and shape are grouped to form cluster based on the tags mapped with image [6]. Based on Wrinkles Features of Human Faces images are used to predicate the age of the person by using different classification algorithm [7]. Gene expression dataset feature selection is so different from above kind. We need to identify the best feature from the dataset using wrapper based feature selection methods.

The feature selection characteristics have been unconnected to the learning method and thus have a better level of generalization..In the case of the wrapper methods, the feature selection was "wrapped" about their learning technique: the feature worth is judged directly by the accuracy of the learning technique. The samples of gene expression data include classifier design and feature selection [8]. The latter identifies the gene subsets which are expressed differentially and are relevant to be able to distinguish the sample classes. The supervised and the unsupervised classifiers had built some models of classification from their microarray data. In the domain of computational intelligence, the gene selection is known as a feature selection. This type of selection of genes has many advantages. It is capable to retain or even increase the classification accuracy. It can reduce the data dimensionality. It is capable of reducing the time taken for computation. It is able to remove the noisy genes or the irrelevant genes. This can bring down the clinical setting cost.

In the recent years, the Swarm Intelligence (SI) is becoming even better-looking for researchers working in the associated research area. This is later classified as a new branch in the evolutionary computing. The SI is defined as to be the measure which introduces the social insect colonies and their collective behavior for designing the algorithms and these are generally effectively applied for solving problems. A classical algorithm found in evolutionary computing was employed for solving optimization problems in the GA. Following this, the SI algorithms will be employed to solve problems for which the Particle Swarm Optimization (PSO), the Cat Swarm Optimization (CSO), the Fast Particle Swarm Optimization (FPSO), the ABC and the Parallel Cat Swarm Optimization (PCSO) are employed. Furthermore, there are many other applications of this algorithm which is based on the SI or the computational intelligence [9].

An ABC algorithm was presented in the year 2005 by Karaboga and a presentation analysis was made in the year 2007. Here, artificial agents are defined and also categorized into 3 different categories that are the employed, the scout and the onlooker bee. Individual bee has a completely different role to play in this procedure: an employed bee stays within the of the food and will provide the source and its neighborhood in its memory; an onlooker gets information of the source of food from the bees which are from the employed bees and further chooses the source of food for gathering 
nectar. The scout is the one that is liable for identifying a new source of nectar [10].

The GA is the search algorithm of the adaptive optimization which simulates all evolutionary ideas in natural selection. There were also some more general steps that are specified which are as below. The Genetic algorithm will now arbitrarily generate its initial population along with the individual chromosomes and they will be assessed using a fitness function. Each individual will look out for some ideal solutions by means of employing GA operators: the selection, the mutation, and crossover. A selection operator selects chromosomes along with some greater adaptive values from its existing population [11]. The mutation operator will alter either one or more values of the gene within the chromosome for the initial stage. A crossover operator is employed for combining two different chromosomes for producing two other new chromosomes that are known as the offspring. There is also a fitness function that is employed for evaluating the chromosome and its quality in the step of evaluation. In the design of the chromosome, there is yet another binary coding system which is used for representing this chromosome. Every bit of this chromosome will represent the gene mask. Another bit with a value ' 1 ' that represents the gene which is chosen and the ' 0 ' will represent a gene which is discarded. The genes having a value ' 1 ' will be chosen and also combined to be the candidate gene subset.

The work further proposed the method of $\mathrm{ABC}$ optimized by Levy search for a bone marrow PC classification with in the data of gene expression.

\section{METHODOLOGY}

Here, GEO Data Sets (GDS) was employed and there is high dimensionality with fewer numbers of features with consideration and preprocessing. A GA based feature selection along with the $\mathrm{ABC}$ and the Levy search are discussed in this section.

\subsection{Dataset}

Gene Expression dataset are searched using various features along with the organism, its keyword and the type of dataset. Two datasets are known as the DS321 and DS4321. There are a total of around 182 samples with 11525 features used within the DS321 dataset. Another set of 64 samples having 21234 features are further used within the DS4321 dataset.

\subsection{Genetic algorithm (GA) Based Feature Selection}

The GA had been employed for choosing binary strings and several authors have been discussing the need of the Genetic algorithm in the feature selection case. Another critical trait of the GA was that it was developed in which inter-dependencies among the bits in the string are explored. Thus it is quite reasonable for these problems in feature selection to look out for their dependencies and choose the ones that are ideal in terms of their contribution to the recognition or classification of performance. For most such cases, the performance is better related to the backward or the forward algorithms of search in linking with the actual number of evolutions for reaching the minimum [12].

The chances of being able to get a feature subset that was optimal during this time the classification accuracy was high when the Genetic algorithm was used for feature selection along with possible considerations and their fitness functions. Taking advantage of this, the scheme of best feature selection which was based on the Genetic Algorithm had been proposed. This wrapper based method in feature selection which was proposed also takes further benefit of a supervised learning algorithm for evaluation of feature subset significance and also utilizes the GA for evaluating the feature subset and its significance in terms of optimizing the process of feature selection. The proposed technique and its exhibition had been tried dependent on the clinical dataset with different classifiers.

The GA is the technique used for shifting to another novel population from that of the present chromosome population by means of natural selection. It likewise has two different administrators that are the hybrid and the transformation. The subparts of the two chromosome are exchanged and then performs a recombination among two different chromosome The mutation, on the other hand, will now randomly change all allele values of certain places within the chromosomes. The GA further assesses the fitness of every individual and this only implies that the results and their superiority had been achieved by fitness function mean. A chromosome which is appropriate will have a higher possibility of having the option to pick the arrangement of the subsequent generation. On the off chance that the chromosome which is the fittest inside the populace can't address the issues, the two elements of crossover and mutation are done. This is repeated continuously until such time an acceptable result is derived.

The algorithm will initially assign a binary search space since the chromosomes as per figure 1 are the bit strings and every bit will represent a feature of its training dataset. There is an initial population that is randomly created assuming the gene value ' 1 ' to represent a certain feature which is to be chosen for evaluation and for maintaining a similar position in the gene value (the same index) and in case it is equal to ' 0 ', this feature will not be chosen and will maintain the same gene value position [13]. Parents will be chosen randomly and their length will remain similar to the actual number of features (in case the total of features is 5 , the chromosomes will contain about 5 genes that denote 5 bits). 


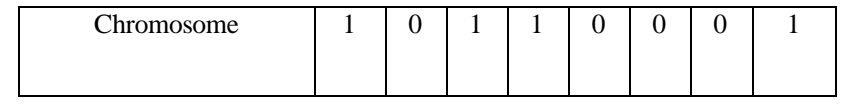

Figure 1: Portrayal of the chromosome for the dataset having Eight Features

The following stage will play out a crossover operation. There are two guardians who are two distinct people (chromosomes) that are joined for shaping another chromosome called child. Such a crossover operation gets done using four logical operations like the XOR operation, the single point crossover operation, the AND operation and the OR operation which is nothing but a sample of operations depicted as per Figure 2 where in the $\mathrm{C} 1$ and $\mathrm{C} 2$ are the children, and the P1 and P2 denote the parents.

\begin{tabular}{|l|l|l|l|l|l|l|l|l|}
\hline $\mathrm{P}_{1}:$ & 1 & 0 & 1 & 0 & 0 & 1 & 1 & 1 \\
\hline $\mathrm{P}_{2}:$ & 1 & 1 & 1 & 1 & 0 & 1 & 1 & 0 \\
\hline $\mathrm{C}_{1}:$ & 1 & 0 & 1 & 0 & 0 & 0 & 1 & 0 \\
\hline $\mathrm{C}_{2}:$ & 1 & 1 & 1 & 1 & 0 & 1 & 1 & 1 \\
\hline
\end{tabular}

(a)

\begin{tabular}{|l|l|l|l|l|l|l|l|l|}
\hline $\mathrm{P}_{1}:$ & 1 & 0 & 1 & 0 & 0 & 1 & 1 & 1 \\
\hline $\mathrm{P}_{2}:$ & 1 & 1 & 1 & 1 & 0 & 0 & 1 & 0 \\
\hline $\mathrm{C}:$ & 1 & 0 & 1 & 0 & 0 & 0 & 1 & 0 \\
\hline
\end{tabular}

(b)

\begin{tabular}{|l|l|l|l|l|l|l|l|l|}
\hline $\mathrm{P}_{1}:$ & 1 & 0 & 1 & 0 & 0 & 1 & 1 & 1 \\
\hline $\mathrm{P}_{2}:$ & 1 & 1 & 1 & 1 & 0 & 0 & 1 & 0 \\
\hline $\mathrm{C}:$ & 0 & 1 & 0 & 1 & 0 & 1 & 0 & 1 \\
\hline
\end{tabular}

(c)

\begin{tabular}{|l|l|l|l|l|l|l|l|l|}
\hline $\mathrm{P}_{1}:$ & 1 & 0 & 1 & 0 & 0 & 1 & 1 & 1 \\
\hline $\mathrm{P}_{2}:$ & 1 & 1 & 1 & 1 & 0 & 0 & 1 & 0 \\
\hline $\mathrm{C}:$ & 1 & 1 & 1 & 1 & 0 & 1 & 1 & 1 \\
\hline
\end{tabular}

(d)

Figure 1: The crossover operation (a) single point (b) AND

$$
\text { (c) XOR (d) OR }
$$

The following third step will play out a mutation operation which can bring about a change the allele values randomly from a crossover on the child along with a new random mute in the fourth position of child (the bit ' 1 '). Figure 2 describes about Mutation operation and the fourth located bit of the child (C) is muted. This next generation (the new chromosome - 1111000111 ) indicates the dataset which consists of a total of about eight features as the chromosome has eight bits and every one of it will represent the feature's index in the dataset. After this, the features that correspond to a chromosome with value ' 0 ' gets rejected and a new subset attained from the dataset which can be f1, f2, f3, f6, f7, f8 in which the $\mathrm{f} 4$ and the $\mathrm{f5}$ will be discarded as their bit position represents 0 .

\begin{tabular}{|l|l|l|l|l|l|l|l|l|}
\hline C: & 1 & 1 & 1 & 1 & 0 & 1 & 1 & 1 \\
\hline Crossover C: & 1 & 1 & 1 & 0 & 0 & 1 & 1 & 1 \\
\hline
\end{tabular}

Figure 3:.Mutation operation with the fourth positioned bit of the child is muted

For the fourth step, the feature subset obtained has been used to calculate the fitness value for validation of a subset which is generated from step 3 . The accuracy of classification is taken to be the fitness value that validates a subset which is generated. For the accuracy of classification (the fitness value) this will have satisfied the condition of termination, the feature subset selected or move to step 2 to 4 that will have to be followed in a way that is iterative until termination condition is satisfied.

\subsection{Artificial Bee Colony (ABC) Based Feature Selection}

In the approach that is proposed, there is an optimization of the $\mathrm{ABC}$ algorithm and this yields an ideal and optimal feature subset for increasing predictive classifier accuracy. The $\mathrm{ABC}$ is further used to be a feature selector for generating a feature subset along with a classifier for the evaluation of the subsets that are produced by the onlooker. Initially, a classifier such as the Ada Boost, the fuzzy or the C4.5 is used for evaluating the dataset. A predictive accuracy of $\left({ }^{x_{i}}\right)$ for each feature $\mathrm{i}$ was then calculated by means of employing a 10-fold cross-validation. After this, an objective $\left(f_{i}\right)$ was computed for the feature from the indiscernibility relation [14].

There was further binary bit string (of a length that was equivalent to the actual number of dataset features) that had been assigned to every employed bee and this represented the feature selection. In the case of a binary string, in case the nth bit is ' 1 ' it only means that the associated feature which is chosen and ' 0 ' means the feature which is not chosen. The features have been considered to be as the feature that is not chosen. There were features that were taken to be the sources of food and this way the population of both the onlooker and the employed bees were found to be same to the actual number of features $(\mathrm{m})$ found in the dataset.

Every employed bee had been assigned along with a new feature and this will evaluate the feature's fitness from the objective $\left(f_{i}\right)$ by making use of the equation (1).

$$
\text { fit }_{i}=1 /\left(1+f_{i}\right)
$$


An onlooker bee will now gain information obtained from an employed bee and will then calculate the chances of choosing features by means of making use of equation (2).

$$
p_{i}=\frac{f i t_{i}}{\sum_{i=1}^{m} f i t_{i}}
$$

After this, the onlooker will duly compute its new solution which is $v_{i}$ by ensuring use of the predictive accuracies belonging to the feature of the employed bee that points to and features what an onlooker bee has chosen. In case a new solution $v_{i}$ is found to be greater than the $x_{i}$, then the employed bee will point to its feature subset containing features it pointed to earlier and also it is the newly chosen feature. In case the $v_{i}$ is not found to be greater than the $x_{i}$ then, employed bees tend to retain the old one and the afresh chosen feature will be ignored. A new solution which is $v_{i}$ will now be computed by means of making use of equation (3).

$$
v_{i}=x_{i}+\varphi_{i}\left(x_{i}-x_{j}\right)
$$

In which the ${ }^{x_{i}}$ denotes a predictive accuracy of the allocated feature made to the employed bee and the ${ }^{x_{j}}$ denotes a predictive accuracy of that of the features chosen by the onlooker. The $\phi_{i}$ denotes the real random numbers that are distributed uniformly within the range $[0,1]$. Like this, every time there is an employed bee which is assigned with a feature subset that is new, the onlooker tends to exploit and then produce another new configuration of feature subset [15].

Once the possible feature subset was generated from the feature, the substance of the nectar gets aggregated towards a subset arrangement which is seen as better. In case the employed bee is not able to show any improvement, it changes into a scout. This scout is then allocated yet another new binary set which is formed using the equation (4).

$$
x_{i}^{j}=x_{\min }^{j}+\operatorname{rand}[0,1]\left(x_{\max }^{j}-x_{\min }^{j}\right)
$$

In which the $x_{\max }^{j}$ and the $x_{\min }^{j}$ will represent the lower, as well as the upper bounds of the population. These bees will continue to execute a similar process for various runs until such time an ideal feature subset is formed. A feature selection based on the ABC is as below:

1. Start the cycle one

2. ABC parameters are initialized

3. Each Individual feature's fitness are evaluated

4. Repeat
5. Built solution by the employed bees

(i) Set feature subset arrangement (binary bit string) to each employed bee

(ii) Generate new bone feature subsets vi

(iii) Send the generated feature subset to the classifier

(iv) Fitness (fiti) of the feature subset is evaluated

(v) Probability (pi) of feature subset solution is computed

6. Built solutions by the onlookers bees(i) Based on Probability pi select a feature

(ii) Calculate vi using $x i$ and $x j$

(iii) Apply greedy selection between $x i$ and vi

7. Determine the scout bee and the neglected solution

8. Evaluate the finest feature subset of the cycle

9. Store the finest feature subset

10. Assign Cycle $=($ Cycle $)+1$

11. Until required number of cycles is attained

12. Perform same searching method of the bees for generating the finest feature subset configurations

\subsection{Proposed ABC - Levy Search Based Feature Selection}

The algorithm ha also produced certain results which have been successful in various benchmark functions. It performs better using a global search with random methods and come across some crisis in the operation and may also get stuck within the local minimum in the case of multimodal functions that are complex. In case the original algorithm fails to show improvement, there is an employed bee that has a source will become the scout bee. After this, the scout bee will be allocated at random in the search area and their boundaries are thus determined [16,17].

Here, the scout bees chose a zone wherein the search space is used with the results that are found until such time this is an outcome of such iterations. There is also a least probability for a food source that is newly chosen to be better on being compared to the Global Min (the best value which is in the current iteration) and this is identified. For this work, in order to make use of the scout bees in a better way, they will have to be able to identify another new source of food by employing Levy Flight distribution along with the effect of the Global Min as opposed to choosing a random source of food. This way, another better solution is attained and the performance of some random searches and the $\mathrm{ABC}$ algorithm is explored and improved.

A detailed review on the manner in which a distribution is performed by making use of the Levy flight [26], is given along with a new state of its particle is as shown in (5)[16]:

$$
X^{t+1}=X^{t}+\alpha \oplus \operatorname{Levy}(\beta)
$$




\begin{tabular}{|l|l|l|}
\hline Classification accuracy & DS4321 & DS321 \\
\hline GA & 0.7143 & 0.7803 \\
\hline ABC & 0.7857 & 0.8092 \\
\hline ABC-Levy & 0.8393 & 0.8439 \\
\hline
\end{tabular}

In which $\alpha$ denotes the size of the step that is related to a problem of interest. In this method, $\alpha$ denotes a random number for the particle dimensions as in (6) [26].

$$
X^{t+1}=X^{t}+\operatorname{random}(\operatorname{size}(D)) \oplus \operatorname{Levy}(\beta)
$$

(6)

A product $\oplus$ denotes multiplications that are entry-wise. There is also a non-trivial scheme to generate $s$ that is a step size summarized as in (7):

$$
s=\operatorname{random}(\operatorname{size}(D)) \oplus \operatorname{Levy}(\beta) \sim 0.01 \frac{u}{|v|^{1 / \beta}}\left(x_{j}^{t}-x_{i}^{t}\right)
$$

Wherein both $v$ and $u$ are drawn from among normal distributions which are (8 \& 9):

$$
u \sim N\left(0, \sigma_{u}^{2}\right) \quad v \sim N\left(0, \sigma_{v}^{2}\right)
$$

With the

$$
\sigma_{u}=\left\{\frac{\Gamma(1+\beta) \sin (\pi \beta / 2)}{\Gamma[(1+\beta) / 2] \beta 2^{(\beta-1) / 2}}\right\}^{1 / \beta}, \sigma_{v}=1
$$

Here the $\Gamma$ denotes a standard Gamma function.

Yet another critical point that needs consideration during the time of performing a distribution by the Levy flights was a value of parameter $\beta$. As mentioned earlier, Yang and Deb had stated that a $\beta$ parameter for different values had provided different results in all trials which had been carried out in the work and this was called a design optimization for a multi-objective cuckoo search and this way it was duly decided as a different $\beta$ parameter can be used for every such benchmark function. Also, the evolutionary algorithms having the adaptive levy mutations were proposed by Chang-Yong Lee. The constant values had been set for parameter $\beta$ conducted by means of a calculation of distribution for every one of these values and choosing another ideal offspring that was produced. Thus, the parameter $\beta$ can affect distribution to a substantial level.

\section{RESULTS AND DISCUSSION}

In this research, the DS321 dataset and DS4321 dataset are used. 182 numbers of samples with 11525 numbers of features are used in DS321 dataset. 64 numbers of samples with 21234 numbers of features are used in DS4321 dataset. The classification accuracy, F measure, specificity, sensitivity, and specificity as shown in tables 1 to 4 and figures 4 to 7 .

Table 1: Classification Accuracy Results of Different Methods

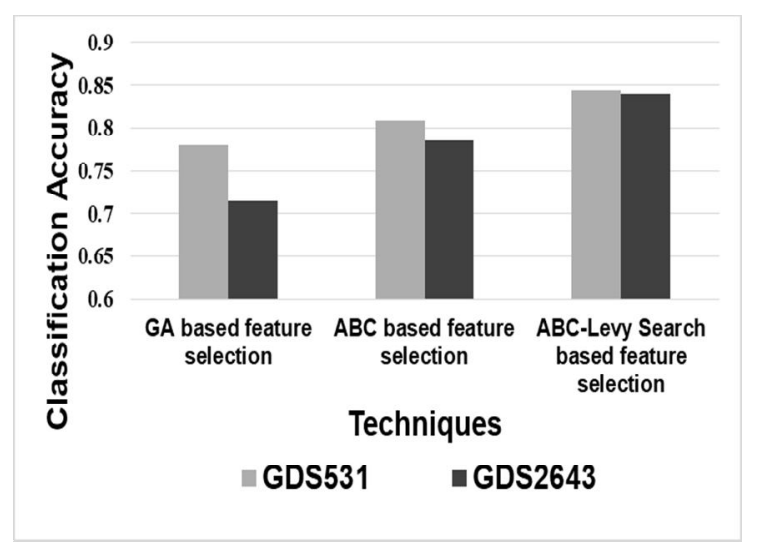

Figure 4: Classification Accuracy Results of Different Methods

From the Figure 4 and Table 1, it very well seen that the ABC-Levy search based feature selection has higher classification accuracy for DS321 dataset by $7.83 \%$ and by 4.19\% when compared with feature selections using GA and ABC. The ABC-Levy search has higher accuracy for DS4321 dataset by $16.09 \%$ and by $6.59 \%$ when compared with feature selections using $\mathrm{GA}$ and $\mathrm{ABC}$.

Table 1: Sensitivity Results of Different Methods

\begin{tabular}{|l|l|l|}
\hline Sensitivity & DS4321 & DS321 \\
\hline GA & 0.6787 & 0.77705 \\
\hline ABC & 0.7632 & 0.80995 \\
\hline ABC-Levy & 0.81825 & 0.83805 \\
\hline
\end{tabular}

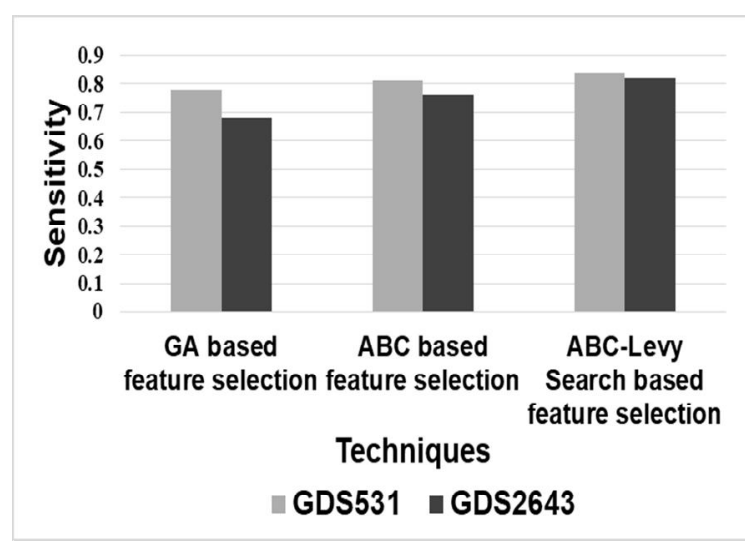

Figure 5: Sensitivity Results of Different Methods 
From the Figure 5 and Table 2, it very well seen that the ABC-Levy search based feature selection has higher sensitivity for DS321 dataset by $7.55 \%$ and by $3.41 \%$ when compared with feature selections using GA and $\mathrm{ABC}$. The ABC-Levy search based feature selection has higher sensitivity for DS4321 dataset by $18.64 \%$ and by $6.96 \%$ when compared with feature selections using $\mathrm{GA}$ and $\mathrm{ABC}$

Table 2: Specificity Results of Different Methods

\begin{tabular}{|l|l|l|}
\hline Specificity & DS4321 & DS321 \\
\hline GA & 0.6787 & 0.77705 \\
\hline ABC & 0.7632 & 0.80995 \\
\hline ABC-Levy & 0.81825 & 0.83805 \\
\hline
\end{tabular}

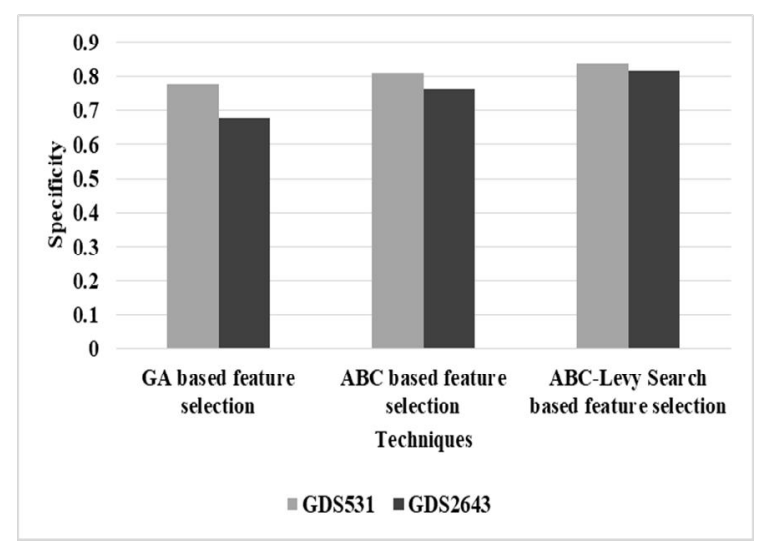

Figure. 4: Specificity Results of Different Methods

From the Figure 6 and Table 3, it very well seen that the ABC-Levy search based feature selection has higher specificity for DS321 dataset by $7.55 \%$ and by $3.41 \%$ when compared with feature selections using GA and ABC. The ABC-Levy search based feature selection has higher specificity for DS4321 dataset by $18.64 \%$ and by $6.96 \%$ when compared with feature selections using GA and $\mathrm{ABC}$.

Table 3: F Measure Results of Different Methods

\begin{tabular}{|l|l|l|}
\hline F measure & DS4321 & DS321 \\
\hline GA & 0.6725 & 0.77705 \\
\hline ABC & 0.7544 & 0.8076 \\
\hline ABC-Levy & 0.81295 & 0.84035 \\
\hline
\end{tabular}

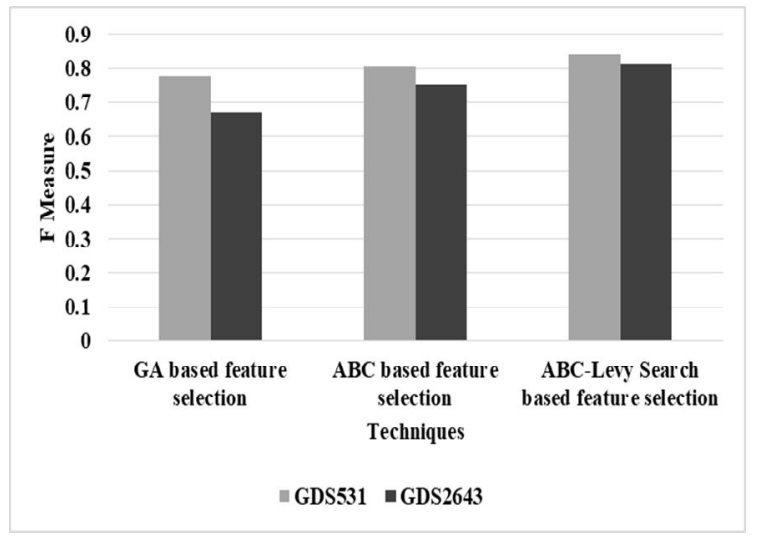

Figure. 5: : F Measure Results of Different Methods

From the Figure 7 and Table 4, it very well seen that the $\mathrm{ABC}$-Levy search based feature selection has higher $\mathrm{f}$ measure for DS321 dataset by $7.82 \%$ and by $3.97 \%$ when compared with feature selections using GA and $\mathrm{ABC}$. The $\mathrm{ABC}$-Levy search based feature selection has higher $\mathrm{f}$ measure for DS4321 dataset by $18.91 \%$ and by $7.47 \%$ when compared with the feature selections using GA and $\mathrm{ABC}$.

\section{CONCLUSION}

The DNA microarrays were used for the purpose of understanding the gene expression and predicting the diseases mainly cancer. There have been several techniques that were proposed in the literature with a limited amount of success. The work further investigates the GA and the $\mathrm{ABC}$ feature selection along with a local search heuristic used for a faster convergence. This is a procedure operating within the binary search spaces. They are used for the automatic determination of their relative importance and to choose good subsets. This $\mathrm{ABC}$ optimization algorithm was an easy and recent probabilistic concept. The levy search is taken to be an algorithmic structure that converges to a close local optimum and will have the potential of detection of a global optimum. The results have proved that the feature selection based on the $\mathrm{ABC}$-Levy search has a higher accuracy of classification for the DS321 dataset by about $7.83 \%$ and by about $4.19 \%$ on being compared to the feature selection based on the Genetic algorithm and the ABC. An ABC-Levy search feature selection has a higher accuracy for the DS4321 dataset by about $16.09 \%$ and by about $6.59 \%$ on being compared with the feature selection based on the Genetic algorithm and the feature selection based on the $\mathrm{ABC}$.

\section{REFERENCES}

[1] L.Y. Chuang, H.W. Chang, C.J. Tu, \& C.H. Yang. Improved binary PSO for feature selection using gene expression data. Computational Biology and Chemistry, 32(1), 29-38,2008 . https://doi.org/10.1016/j.compbiolchem.2007.09.005

[2] Q. Shen, W.M. Shi, \& W. Kong. Hybrid particle swarm optimization and tabu search approach for 
selecting genes for tumor classification using gene expression data. Computational Biology and Chemistry, 32(1), 53-60,2008.

[3] F. Zhan, E. Tian, K. Bumm, R. Smith, B. Barlogie, \& J. Shaughnessy. Gene expression profiling of human plasma cell differentiation and classification of multiple myeloma based on similarities to distinct stages of late-stage B-cell development. Blood, 101(3), 1128-1140, 2003. https://doi.org/10.1182/blood-2002-06-1737

[4] B.G. Barwick, C.D. Scharer, R.J. Martinez, M.J. Price, A.N. Wein, R.R. Haines, \&J.M Boss. B cell activation and plasma cell differentiation are inhibited by de novo DNA methylation. Nature communications, 9(1), 1900.2018.

https://doi.org/10.1038/s41467-018-04234-4

[5] S.M. Uma. A Hybridization of Genetic-Firefly Algorithm Algorithm Technique for Feature Selection in Micro Array Gene Data. International Journal of Innovations \& Advancement in Computer Science (IJIACS), 7 (4), 70-81,2018.

[6] Saravana Balaji B, Krishna Kumar V, Ahmed Najat Ahmed. Semantically enriched Tag clustering and image feature based image retrieval.International Journal of Advanced Trends in Computer Science and Engineering,https://doi.org/10.30534/ijatcse/2019/2381. 22019

[7] Dr. Kachapuram Basava Raju1, Miryala Sukumar ,Potturi Sailesh,Alampally Anusha. Automated Age Prediction Using Wrinkles Features of Human Faces. International Journal of Advanced Trends in Computer Science andEngineering,8(1.2).2019

[8] Dr. Kachapuram Basava, Raju,Miryala,Sukumar Potturi Sailesh Alampally Anusha M.S. Mohamad, S. Omatu, S. Deris, \& M.A. Yoshioka.Modified binary particle swarm optimization for selecting the small subset of informative genes from gene expression data. IEEE Transactions on information Technology in Biomedicine, 15(6), 813-822,2011.

[9] P.W. Tsai, J.S. Pan, B.Y. Liao, \& S.C. Chu. Enhanced artificial bee colony optimization. International Journal of Innovative Computing, Information and Control, 5(12), 5081-5092,2009.

[10] D. Karaboga, \& B. Akay. Artificial bee colony (ABC), harmony search and bees algorithms on numerical optimization. In Innovative production machines and systems virtual conference, 2009.

[11] S. Li, W. Wu, \&M. Tan.Gene. selection using hybrid particle swarm optimization and genetic algorithm. Soft Computing, 12(11), 1039-1048,2008. https://doi.org/10.1007/s00500-007-0272-X

[12] O. H. Babatunde, L. Armstrong, J. Leng, \& D. Diepeveen.A genetic algorithm-based feature selection. International Journal of Electronics Communication and Computer Engineering, 5 (4), $899-905,2014$
[13] D.A.A.G. Singh, E.J. Leavline, R. Priyanka, \& P.P. Priya. Dimensionality reduction using genetic algorithm for improving accuracy in medical diagnosis. International Journal of Intelligent Systems and Applications, 8(1), 67,2016.

[14] T. Ragunthar, S. Selvakumar. A wrapper based feature selection in bone marrow plasma cell gene expression data, Cluster Computing, issue 6,2019. https://doi.org/10.1007/s10586-018-2094-2

[15] B. Subanya, \& R. Rajalaxmi. Artificial bee colony based feature selection for effective cardiovascular disease diagnosis. International Journal of Scientific \& Engineering Research, 5(5), 606-612,2014.

[16] S. Palanisamy, \& S. Kanmani.Artificial bee colony approach for optimizing feature selection. Journal of Computer Science Issues, 9(3), 432-438,2012.

[17] H. Hakli, \& H. Uguz.Levy flight distribution for scout bee in artificial bee colony algorithm. Lecture Notes on Software Engineering, 1(3), 254, 2013. https://doi.org/10.7763/LNSE.2013.V1.55 